\title{
Ansatz zur Bestimmung der Unsicherheit und Prognosefähigkeit eines FEM-Simulationsmodells einer kleinen Mehrfachfixpunktzelle für Kalibrierung von Berührungsthermometern
}

Approach to determining the uncertainty and predictive capability of a FEM simulation model of a small multiple fixed-point cell for calibration of contact thermometers

DOI 10.1515/teme-2018-0027

Zusammenfassung: Die Definition und Variation von Eingangsparametern eines Modells erlaubt nach einer Stichprobenberechnung die Auswertung von verteilten Ausgangsparametern. Sie werden als Reproduzierbarkeit dieser Berechnung angesehen. Für ein thermisches FEMModell einer kleinen Mehrfachfixpunktzelle für die Kalibrierung von Berührungsthermometern wird nach dieser Prozedur und nach einem Vergleich (Validierung) mit der Reproduzierbarkeit experimentell ermittelter Ergebnisse mittels einer Validierungsmetrik ein Vertrauensintervall berechnet, in dem Prognosen mit dem Modell an einer nicht validierten Stelle durchgeführt werden könnten. Dieser Ansatz wird in diesem Beitrag vorgestellt.

Schlüsselwörter: Fixpunktzelle, Area-Metrik-Methode, Validierung, Prognosefähigkeit.

Abstract: The definition and variation of input parameters of a model allows after a sample calculation the evaluation of his distributed output parameters. These are considered as reproducibility of this calculation. A thermal FEM model of a small multiple fixed-point cell for calibration of contact thermometers was simulated according to this procedure and validated with the reproducibility of measurements using a validation metric. After this a confidence interval in which predictions with the model at one not validated point could be carried out, was found. This approach is presented in this article.

\footnotetext{
*Korrespondenzautor: Sebastian Marin, Institut für Prozessmessund Sensortechnik, TU IImenau, Gustav-Kirchhoff-Straße 1, 98693 Ilmenau, E-Mail: sebastian.marin@tu-ilmenau.de Thomas Fröhlich, Institut für Prozessmess- und Sensortechnik, TU Ilmenau, Gustav-Kirchhoff-Straße 1, 98693 IImenau, E-Mail: thomas.froehlich@tu-ilmenau.de
}

Keywords: Fixed-point cell, area metric method, validation, predictive capability.

\section{Einleitung}

Modelle kommen zum Einsatz, um einen Versuch die Realität zu Beschreiben und daraus Erkenntnisse zu gewinnen. Sie sind ein mathematisches Konstrukt eines physikalischen Vorgangs und basieren wegen der Annahmen, die bei der Modellerstellung getroffen werden, unter anderem auf Vermutungen. Dies bedeutet, dass Modelle eine Abweichung zur Realität enthalten. Diese wird festgestellt bzw. kompensiert, indem sie unter bestimmten Voraussetzungen mit experimentell ermittelten Ergebnissen verglichen werden, also eine sogenannte Validierung durchgeführt wird. Dies wird im Allgemeinen auf vier unterschiedliche Arten oder Methoden gemacht. Bei der ersten Methode werden die Ergebnisse graphisch dargestellt, eine qualitative Validierung. Ein Beispiel davon in der thermischen Ebene wäre der Vergleich der Temperaturverteilungen einer thermographischen Abbildung mit Ergebnissen einer FEM-Simulation. Die zweite und am weiteste verbreitete Validierungsmethode ist ein diskreter Vergleich der Ergebnisse. Hierfür werden die Resultate an einem gemessenen Punkt oder einer gemessenen Kurve mit einem simulierten Punkt bzw. einer simulierten Kurve verglichen. In der dritten Methode werden die Simulationsergebnisse in einem Bereich der Messergebnisse verglichen, wobei dieser von der Messunsicherheit abgegrenzt wird. Abschließend findet in der vierten Methode ein probabilistischer Vergleich der Ergebnisse statt, wobei sowohl Mess- als auch Simulationsergebnisse mit Unsicherheiten definiert werden müssen [31]. In diesem Fall werden zwei Wahrscheinlichkeitsvertei- 
lungen miteinander verglichen, was in der Regel nach drei Lösungswegen erfolgt [10]: Statistische Tests (KolmogorowSmirnow-Test [44], Kulback-Leibler-Divergenz), Satz von Bayes oder Frequentist's Metric (Vergleich zentraler Tendenzen) [31].

Eine zusätzliche Validierungsmethode, die sogenannte Area-Metrik, basiert auf den Minkowsky-Abstand erster Ordnung und wurde von Ferson vorgeschlagen [10, 31]. Dabei wird die gesamte Differenzfläche $\left(A_{\mathrm{t}}\right.$ in Abb. 1$)$ zwischen den Verteilungsfunktionen als Übereinstimmungsmaß der Ergebnisse angegeben. Eine kleinere Fläche entspricht eine bessere Übereinstimmung der beiden kumulierten Verteilungen. Die Interpretation des Übereinstimmungsmaßes gestaltet sich als schwierig, da die Differenzfläche skalenabhängig ist und deswegen können auch große Werte eine kleine Fläche und dementsprechend eine gute Übereinstimmung der Ergebnisse darstellen.

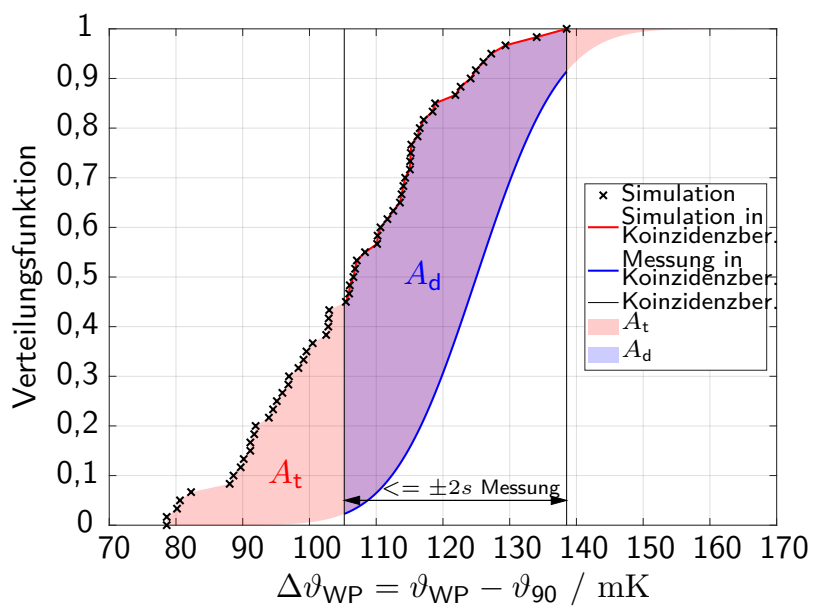

Abb. 1: Graphische Darstellung der Berechnung des Übereinstimmungsmaßes am Beispiel der Fixpunkttemperatur von $\mathrm{Zn}$.

Der Einsatz von validierten Modellen unter nicht validierten Voraussetzungen, um Prognosen oder Optimierungen durchzuführen, ist eine übliche Praxis. Dabei wird aber in der Regel nicht in Betracht gezogen, ob das Modell dafür geeignet ist. In diesem Beitrag wird die Fähigkeit eines thermischen FEM-Simulationsmodells untersucht, eine Prognose unter Berücksichtigung ihrer Modellunsicherheiten zu machen. Das dafür verwendete System ist eine kleine Mehrfachfixpunktzelle, k-MFFPZ, für die zur Internationalen Temperaturskala von 1990, ITS-90 ( $\left.\vartheta_{90}\right)$ [35] rückführbare Kalibrierung von Berührungsthermometern.

\section{Kleine Mehrfachfixpunktzelle für die Kalibrierung von Berührungsthermometern}

Für die Kalibrierung von Berührungsthermometern werden ein Medium mit einer homogenen Temperatur und die bekannte Temperatur dieses Mediums benötigt. Die zwei Voraussetzungen werden durch einen Ausgleichsblock oder eine Flüssigkeit und einen kalibrierten Temperaturfühler bei einer Vergleichskalibrierung oder durch ein reines Material (Fixpunktmaterial) und seine Fixpunkttemperatur (Schmelz- oder Erstarrungstemperatur) in einer Fixpunktzelle bei einer Fixpunktkalibrierung bereitgestellt. Die Fixpunkttemperatur ist unter bestimmten Bedingungen konstant und sehr gut reproduzierbar. Sie wird aus der Phasenumwandlung des Materials zwischen ihren Agregatzuständen, in Form eines Temperaturplateaus, ausgewertet. Auf diesem Prinzip beruht die Internationale Temperaturskala von 1990, ITS-90 [35]. Diese Fixpunkte werden in speziellen Öfen induziert, was die Anwendung in der Praxis erschwert und unzugänglich machen kann. Mit dem Ziel, den Kalibrieraufwand unter Beibehaltung einer höhen Reproduzierbarkeit der Fixpunkttemperatur $\mathrm{zu}$ reduzieren, wurde eine kleine-Mehrfachfixpunktzelle (k-MFFPZ) entwickelt [25]. Sie wurde als Kalibrierreferenz für den Referenzfühler eines Temperatur-Blockkalibrators (T-BK) im Bereich von $80^{\circ} \mathrm{C}$ bis $430{ }^{\circ} \mathrm{C}$ konzipiert [16] Die Zelle wurde modular gebaut und besteht aus drei getrennten Graphittiegeln, gefüllt mit den reinen Stoffen Indium (In, Fixpunkttemperatur $\vartheta_{\mathrm{FP}}=156,5985^{\circ} \mathrm{C}$ ), Zinn $\left(\mathrm{Sn}, \vartheta_{\mathrm{FP}}=231,928^{\circ} \mathrm{C}\right)$ und $\operatorname{Zink}\left(\mathrm{Zn}, \vartheta_{\mathrm{FP}}=419,527^{\circ} \mathrm{C}\right)$ und einem vierten externen Tiegel, in den alle andere konzentrisch eingesteckt werden (Abb. 2). Auf diese Weise ist es möglich, ein Thermometer in einem Messzyklus an drei unterschiedlichen Temperaturen zu kalibrieren.

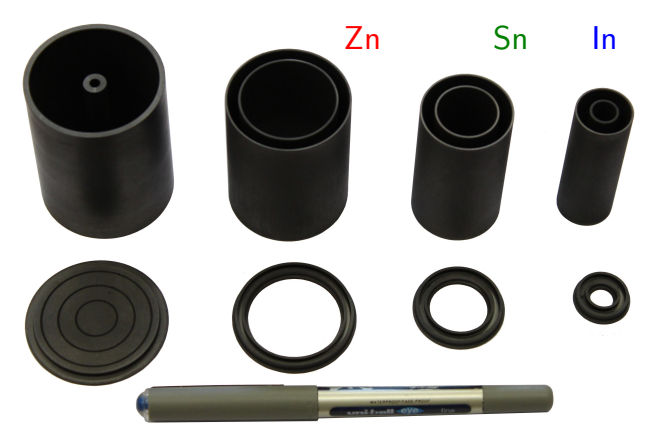

Abb. 2: Modulare Graphittiegel der kleinen Mehrfachfixpunktzelle. 
Die Ermittlung der Fixpunkttemperatur (Bestimmung eines Temperaturwerts auf dem Plateau) erfolgt nach unterschiedlichen praktischen Verfahren [19]. Im hier beschrieben Fall erfolgte die Auswertung nach der Wendepunktmethode. Hierfür wird auf das Schmelzplateau eine Kurve dritter Ordnung gelegt und ihr Wendepunkt, wird als $\vartheta_{\text {FP }}$ ausgewertet (Abb. 3). Erstarrungsplateaus wurden nicht weiterverfolgt, weil sich ihre Anwendung im T-BK als nicht praktikabel erwiesen habe.

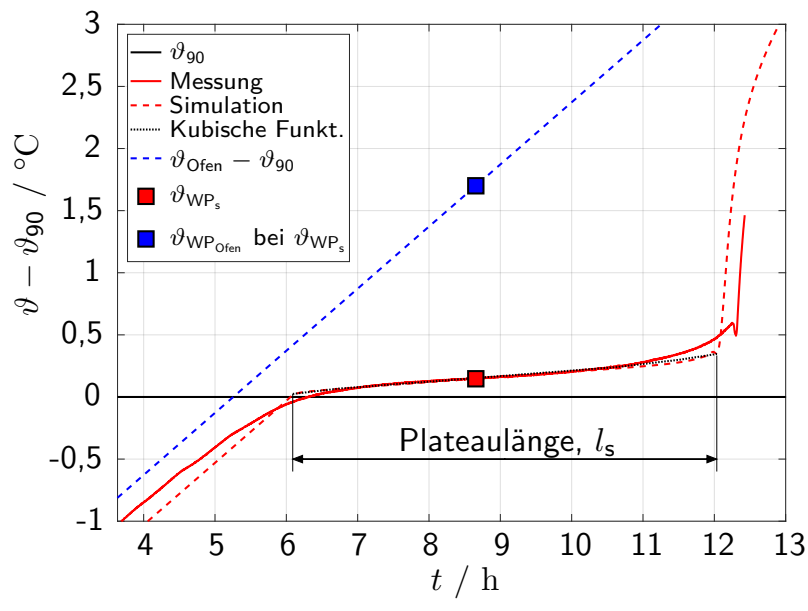

Abb. 3: Simulierte und gemessene Phasenumwandlung von $\mathrm{Zn}$ mit Kennzeichnungen für ihre Auswertung.

Wegen der gleichzeitigen Entwicklung des T-BK und der k-MFFPZ wurde die Langzeituntersuchung der Zelle in einem separaten Aufbau in einem senkrecht positionierten Rohrofen durchgeführt (Abb. 5, links), womit angestrebt wurde, dass die thermische Eigenschaften der k-MFFPZ im T-BK erhalten bleiben. Die Reproduzierbarkeiten der Fixpunkttemperaturen $\left(\vartheta_{\mathrm{WP}_{\mathrm{s}}}\right.$ in Abb. 3), der Plateaulänge ( $l_{\mathrm{s}}$ in Abb. 3) und der Plateausteigung $\left(m_{\mathbf{s}}\right)$ abhängig von der für die Messungen eingestellten Ofentemperatur $\left(\vartheta_{\mathrm{WP}_{\text {ofen }}}\right.$ bei $\vartheta_{\mathrm{WP}_{\mathrm{s}}}$ in Abb. 3) sowie die Fixpunkttemperaturen mit den dazugehörenden Messunsicherheiten unter adiabatischen Bedingungen $\left(\vartheta_{\mathrm{FP}}\right)$ wurden für das System experimentell ermittelt [26]. Im FEM-Simulationsmodell wurden Eingangsparameter festgelegt, die mit ihrer Variation zur Streuung der Simulationsergebnisse beitragen könnten (u. a. Annahmen bei der Modellvereinfachung und Definition der Randbedingungen, geometrische Größen, Stoffeigenschaften und Simulationsoptionen wie Konvergenztoleranzen und Zeitschritte). Die gestreuten Simulationsergebnisse wurden mit den experimentell ermittelten Reproduzierbarkeiten validiert und daraus wurde ein Vertrauensintervall (Prognosefähigkeit) für das Modell gefunden, in dem es für Prognosen unter nicht validierten Voraussetzungen eingesetzt werden könnte. Zuletzt wurden die Fixpunkttemperaturen unter adiabatischen Bedingungen mit Berücksichtigung des gefundenen Vertrauensintervalls verglichen.

Der Vergleich zwischen gestreuten Mess- und Simulationsergebnissen wurde anhand einer Anpassung der Validierungsmetrik nach Ferson [10] durchgeführt. Dafür wurde ein Verhältnis von Flächen $\left(A_{\mathrm{d}}\right.$ und $A_{\mathrm{t}}$ in Abb. 1 und Gleichung (1)) zwischen den Verteilungsfunktionen im Koinzidenzbereich von bis zu 95\% des Messergebnisses als Übereinstimmungsmaß $\left(P_{\mathrm{F}_{\mathrm{x}}}\right.$ in Gleichung (1)) implementiert. Damit wird die Interpretation erleichtert, da das Übereinstimmungsmaß normiert wird. Eine große Fläche ohne Koinzidenzbereich, bedeutet eine 0-prozentige Übereinstimmung und keine Fläche entspricht einer 100prozentigen Übereinstimmung der Mess- und Simulationsergebnisse.

$$
P_{\mathrm{F}_{\mathrm{x}}}=\left(\frac{A_{\mathrm{d}}}{A_{\mathrm{t}}}\right) \cdot 100 \%
$$

\section{Simulationsmodell}

Das Simulationsmodell der k-MFFPZ wurde als ein vereinfachtes zwei-dimensionales axialsymmetrisches Modell (Abb. 5 rechts) des inneren Messaufbaus (Abb. 5 links) in ANSYS konstruiert und simuliert. Die Heizung im Rohrofen wurde durch freie Konvektion als Randbedingung definiert. Dies erfolgte mit einer Heizrampe von $0,5 \mathrm{~K} \mathrm{~h}^{-1}$. Schnellere Heizraten oder sprungartige Temperaturänderungen erschweren die Konvergenz der Simulation. Das Ofentemperaturprofil wurde experimentell an 3 Punkten ( $p_{1}, p_{2}$ und $p_{3}$ Abb. 5 links und Abb. 10 rechts) ermittelt. Luftspalten bis zu einer Dicke von $0,25 \mathrm{~mm}$ zwischen den Bauteilen wurden nicht als Körper sondern als temperaturabhängige thermische Widerstände definiert. Diese werden als das Verhältnis der Wärmeleitfähigkeit der Luft zu der Dicke der Luftspalte eingetragen. Für die simulierten Luftkörper zwischen der Fixpunktzelle und dem Temperatursensor bzw. den Fixpunktmaterialien und der Fixpunktzelle (Abb. 5) wurden temperaturabhängige Wärmeleitfähigkeiten der Luft unter Berücksichtigung der Strahlung eingesetzt [25]. Die Unabhängigkeit der Simulationsergebnisse sowohl von der Vernetzung als auch von den Konvergenzkriterien [1] wurde separat untersucht und ist gewährleistet (Abb. 4). Dieser letzte Faktor spielt eine sehr große Rolle, da die Plateaulänge und dementsprechend die Plateausteigung und die Fixpunkttemperatur davon abhängig sind (sie korrelieren miteinander Abb. 8). 


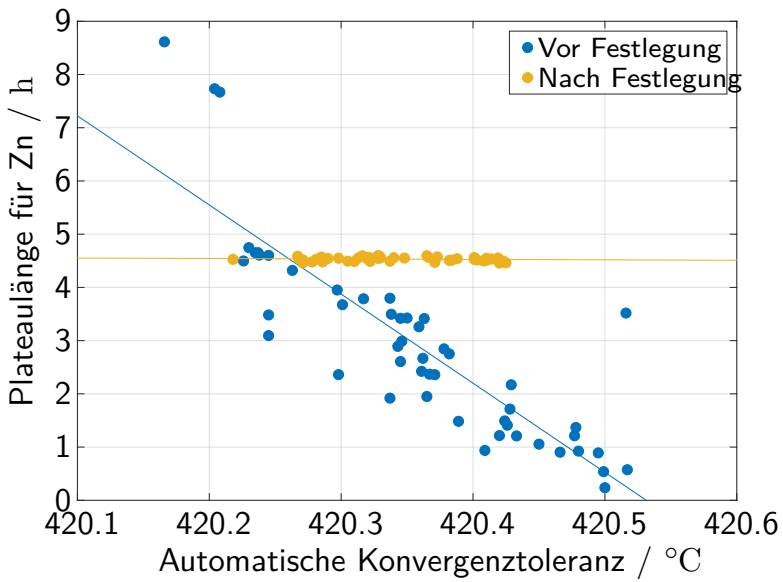

Abb. 4: Plateaulänge für zwei unterschiedliche Durchläufe mit einer Stichprobe von jeweils 50 Berechnungen vor und nach der Festlegung der Toleranzen der Konvergenzkriterien am Beispiel von Fixpunktmaterial Zn.

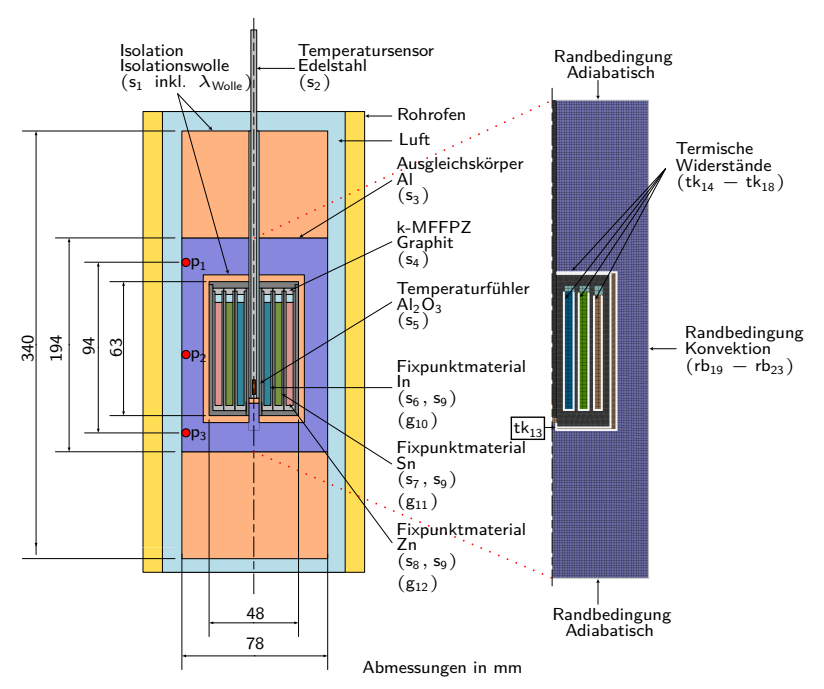

Abb. 5: Schematische Darstellung und FEM-Simulationsmodell des Messaufbaus mit der integrierten kleinen-

Mehrfachfixpunktzelle mit Kennzeichnung der Modellparameter $\left(s_{\mathrm{x}}, 9\right.$ Stoffeigenschaften, $g_{\mathrm{x}}, 3$ geometrische Größen $t k_{\mathrm{x}}, 6$ thermische Kontakte, $r b_{\times}, 5$ Randbedingungen).

\section{Simulationsablauf}

In einer thermischen Simulation wird die Wärmeleitungsdifferenzgleichung (Gleichung (2)) gelöst [17]. In ihr werden geometrischen Größen $(x, y, z)$, Randbedingungen $(\dot{q})$ und Stoffeigenschaften ( $\lambda$ Wärmeleitfähigkeit, $\rho$ Dichte, $c$ spezifische Wärmekapazität), sogenannte Eingangspara- meter für die Berechnung der Temperatur $(\vartheta)$ benutzt.

$$
\frac{\partial}{\partial x}\left(\lambda \frac{\partial T}{\partial x}\right)+\frac{\partial}{\partial y}\left(\lambda \frac{\partial T}{\partial y}\right)+\frac{\partial}{\partial z}\left(\lambda \frac{\partial T}{\partial z}\right)+\dot{q}=\rho c \frac{\partial T}{\partial t}
$$

Den Eingangsparameter (insgesamt 23) wurden Wahrscheinlichkeitsverteilungen zugewiesen und Simulationen mit dem Modell wurden mehrfach durch Stichprobenberechnung nach dem Flussdiagramm (Abb. 6) mit der Software optiSLang [9] durchgeführt. Die Software generiert den Parameterraum (systematisch nach der ALHSMehode [44]), berechnet Korrelationen (Abb. 8) zwischen den Parametern und filtert nach einer Sensitivitätsanalyse nur die wichtigsten heraus, mit denen Antwortflächen und damit reduzierte Modelle berechnet werden. Die Qualität der geschätzten Antwortflächen und die Relevanz der einzelnen Eingangsparameter auf den Ausgangsparameter wird mit einem sogenannten Coefficient of Prognosis (CoP) ausgedrückt. Ein CoP nahe 100\% bedeutet, dass das mit den Antwortflächen beschriebene, reduzierte Modell ausreichend das FE-Modell widerspiegelt (Tab. 1) und die herausgefilterte Parametern tatsächlich die relevanten sind. Darüber hinaus erlaubt optiSLang die Schnittstellen zu den anderen benutzten Programmen.

\begin{tabular}{cccc}
\hline \multirow{2}{*}{ Ausgangsparameter } & \multicolumn{3}{c}{ Fixpunktmaterial } \\
\cline { 2 - 4 } & In & Sn & Zn \\
\hline$\vartheta_{\mathrm{WP}_{\mathrm{s}}}$ & 97 & 97 & 96 \\
$m_{\mathbf{s}}$ & 98 & 97 & 91 \\
$l_{\mathrm{s}}$ & 96 & 96 & 93 \\
\hline
\end{tabular}

Tab. 1: Gesamtes Coefficient of Prognosis (CoP) der ausgewerteten Ausgangsparametern.

Ein Simulationsschritt bestand aus einer statischthermischen-Berechnung, die die Ausgangstemperaturverteilung für eine transient-thermische-Berechnung ergab. Daraus wurde die durch den Temperaturfühler (Abb. 5 links) erfasste Temperatur in Matlab ausgewertet und die simulierten Fixpunkttemperatur, $\vartheta_{\mathrm{WP}_{\mathrm{s}}}$, Plateaulänge $l_{\mathrm{s}}$ und Plateausteigung $m_{\mathbf{s}}$ als Ausgangsparameter einer Simulation ermittelt.

Nach der Sensitivitätsanalyse (rechteckverteilte Eingangsparameter Abb. 8) erfolgte eine Reduktion der Eingangsparameter. Damit wurde eine neue Stichprobenberechnung durchgeführt, mit der festgestellt wurde, dass es sich tatsächlich um die sensitivsten handelt. Ihre Mittelwerte wurden nacher so optimiert, dass die 3 Ausgangsparameter im Bereich der Messergebnisse liegen (erste Validierung). Die gefundenen Mittelwerte wurden als Mittelwerte einer Normalverteilung definiert, denen ei- 


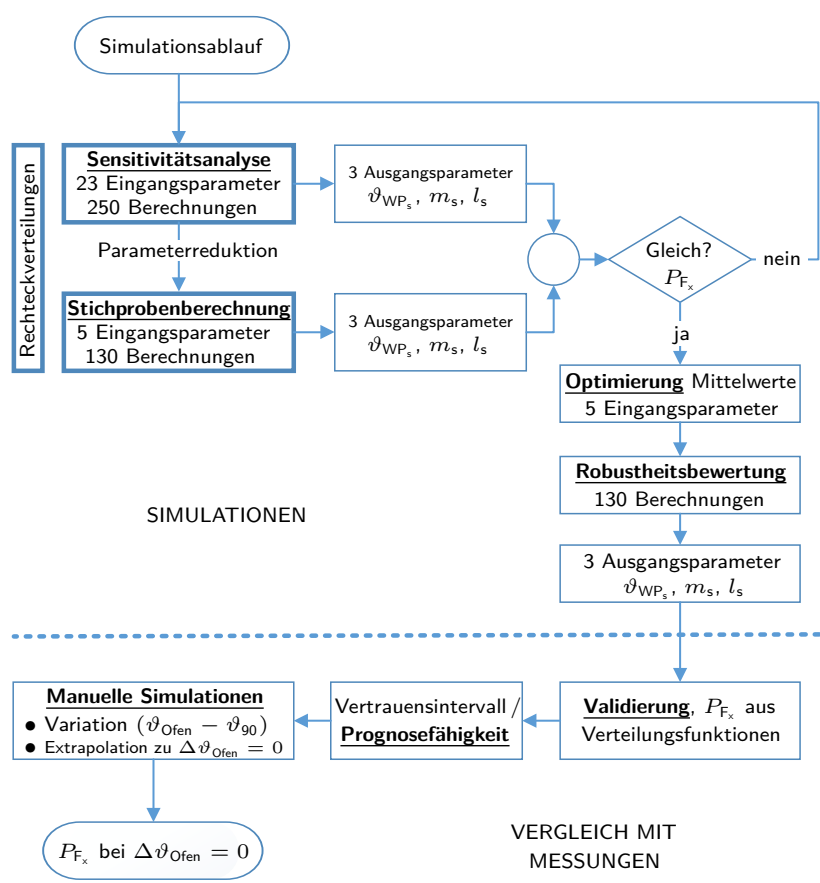

Abb. 6: Flussdiagramm des gesamten Simulationsablaufs.

ne Standardabweichung von $5 \%$ zugewiesen wurde. Dieser Wert entspricht der maximalen relativen Abweichung gut bekannte Stoffeigenschaften (Abb. 10 links). Zusätzliche 130 Simulationen wurden in einer sogenannten Robustheitsbewertung (Normalverteilte Eingangsparameter) durchgeführt. Danach erfolgte die Validierung der Ergebnisse (Abb. 9) nach der Area-Metrik Methode, diese die in Abb. 1 und Gleichung (1) vorgestellt wurde.

Die Größe des Parameterraumes scheint zunächst sehr gering für die Anzahl der Eingangsparameter zu sein. Dies wird aber ausgeschlossen, da die berechneten Antwortflächen relativ linear sind (Abb. 7), die Eingangsparameter nicht miteinander korrelieren (Abb. 8) und die Coefficient of Prognosis $(\mathrm{CoP})$ sehr hoch für die drei Fixpunktmaterialien sind (Tab. 1).

\begin{tabular}{ccccc}
\hline \multirow{2}{*}{ Ausgangsparameter } & \multicolumn{3}{c}{ Fixpunktmaterial } & \\
\cline { 2 - 4 } & In & Sn & Zn & \\
\cline { 1 - 4 }$\vartheta_{\mathrm{WP}_{\mathrm{s}}}$ & 53 & 81 & 70 & Vertrauensintervall / \\
$m_{\mathrm{s}}$ & 69 & 73 & 64 & Prognosefähigkeit \\
$l_{\mathrm{s}}$ & 59 & 69 & 63 & \\
\hline $\bar{x}$ & 61 & 75 & 67 & 67 \\
$s$ & 5 & 3 & 2 & 6 \\
\hline
\end{tabular}

Tab. 2: Prozentuale Übereinstimmung der Validierung der Messund Simulationsergebnissen nach $P_{\mathrm{F}_{\mathrm{x}}}$ in Gleichung 1.

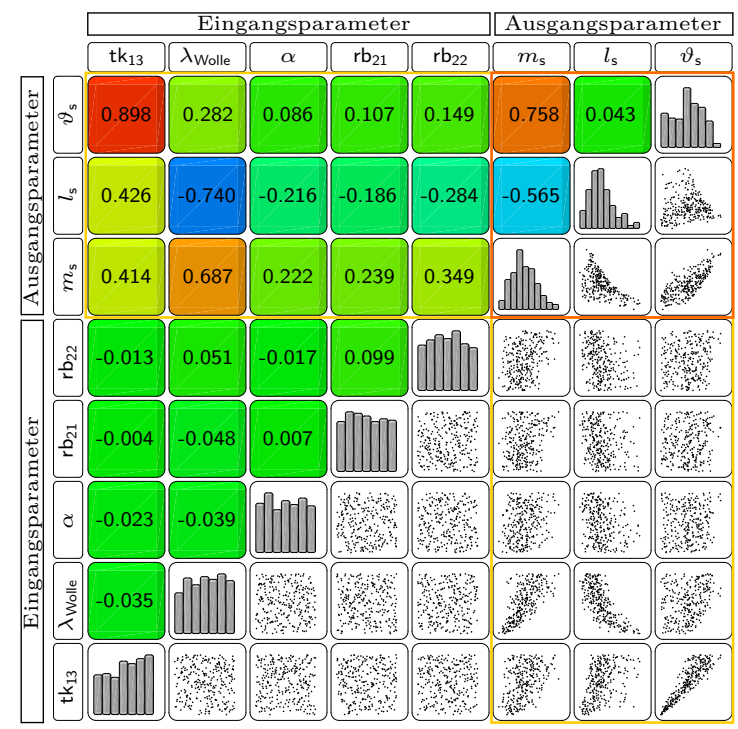

Abb. 7: Korrelationmatrix mit den 5 Sensitivsten Eingangsparametern und die 3 Ausgangsparameter am Beispiel der Sensitivitätsanalyse für Sn. Blau entspricht einer Korrelation von -1 , Rot von 1 und Grün von 0. Orange eingeschlossen sind die Korrelationen der Ausgangsparametern miteinander, gelb eingeschlossen sind die Korrelationen der Ein- auf die Ausgangsparameter. Jeder Punkt in einem Streudiagramm entspricht ein Simulationsschritt.

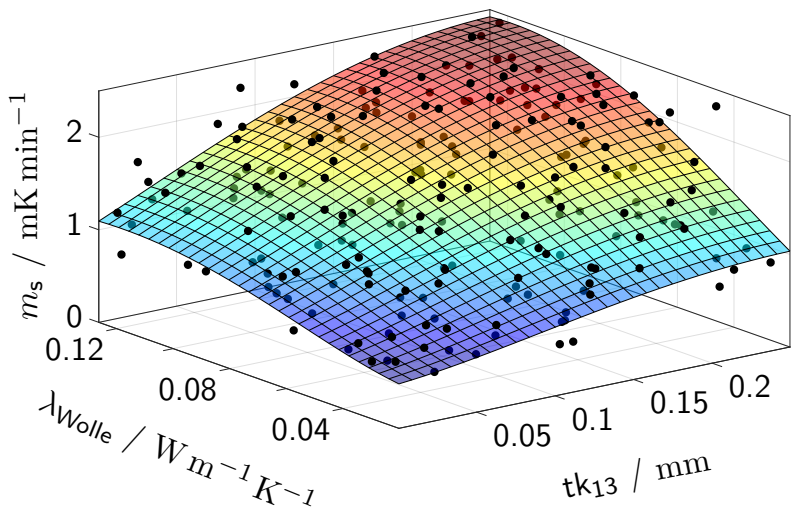

Abb. 8: Antwortfläche für die zwei sensitivsten Parameter der Plateausteigung von In. Die schwarze Punkte stellen die Simulationsergebnisse dar.

Das Vertrauensintervall für die Prognosefähigkeit des Modells wurde nach der Validierung (Abb. 9) aus dem Mittelwert der drei ausgewerteten Ausgangsparametern aller drei Fixpunktmaterialien berechnet (Tab. 2). Es wurde von einer Rechteckverteilung ausgegangen, weil pro Fixpunktmaterial bzw. Modell ausschließlich nur drei Werte vorliegen. Die Ofentemperatur wurde im Bereich von $\vartheta_{\mathrm{WP}_{\text {ofen }}} \pm 250 \mathrm{mK}$ (Abb. 9) als konstant für die Validierung der Reproduzierbarkeiten angenommen, weil es dem 


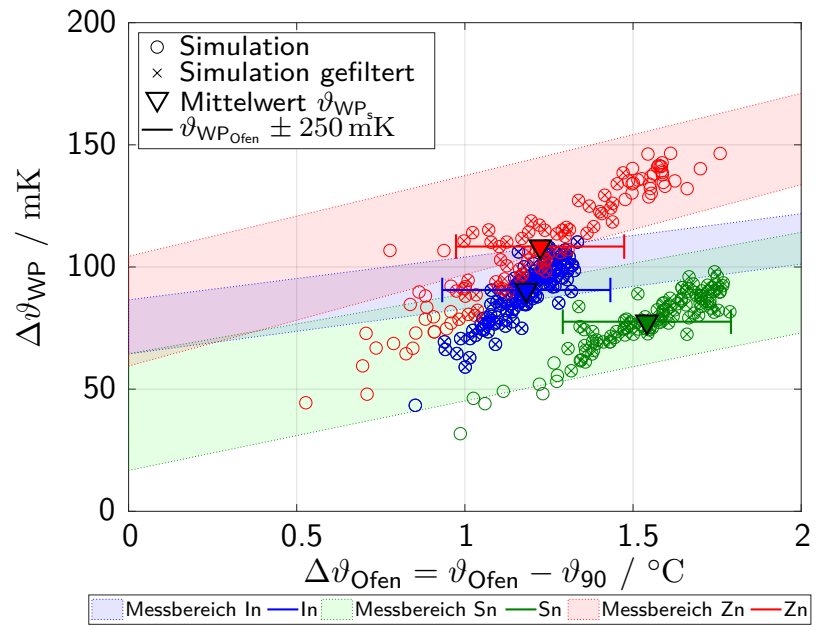

Abb. 9: Validierung der Simulationsergebnisse gegen die Reproduzierbarkeiten der Messergebnissen am Beispiel der Fixpunkttemperatur der drei Fixpunktmaterialien.

Bereich der Reproduzierbarkeit der gemessenen Ofentemperatur entspricht.

\section{Unsicherheitsbestimmung der simulierten Fixpunkttemperatur}

Die Unsicherheit der simulierten Fixpunkttemperatur $\left(\vartheta_{\mathrm{FP}_{\mathrm{s}}}\right)$ unter adiabatischen Bedingungen $\left(\vartheta_{\text {Ofen }}-\vartheta_{90}=0\right.$ in Abb. 9) setzt sich nach folgendem Ansatz zusammen.

$$
\begin{aligned}
& \vartheta_{\mathrm{FP}_{\mathrm{s}}}=\vartheta_{\text {fit }} \pm\left(\delta \vartheta_{\text {fit }}+\delta \vartheta_{\mathrm{WP}_{\mathrm{s}}}+\delta \vartheta_{\text {Modell }}+\delta \vartheta_{\text {stochastisch }}\right) \\
& \delta \vartheta_{\text {stochastisch }}= k_{\mathrm{P}_{\mathrm{F}_{\mathrm{x}}}}\left(\delta \vartheta_{\text {Geometrie }}+\delta \vartheta_{\text {therm. Kontakte }}\right. \\
&\left.+\delta \vartheta_{\mathrm{RB}}+\delta \vartheta_{\text {Stoff }}+\delta \vartheta_{\text {numerisch }}\right)
\end{aligned}
$$

Wobei:

$\vartheta_{\text {fit }}$ : durch Ausgleichsrechnung auf adiabatische Bedingungen extrapolierte Fixpunkttemperatur.

$\delta \vartheta_{\text {fit }}$ : Vertrauensintervall der Ausgleichsrechnung bei adiabatischen Bedingungen.

$\delta \vartheta_{\mathrm{WP}_{\mathrm{s}}}$ : Differenz der approximierten Kurve dritter Ordnung zur der simulierten bei $\vartheta_{\mathrm{WP}_{\mathrm{s}}}$ (Abb. 3).

$\delta \vartheta_{\text {Modell}}:$ Modellvereinfachungsfehler. Wurde durch Vergleich von Simulationsergebnissen mit einem detaillierten Modell ermittelt.

$k_{\mathrm{P}_{\mathrm{F}}}$ : Vertrauensintervall oder Prognosefähigkeit des Modells (Tab. 2). Dies ist abhängig von Geometrie$\left(\delta \vartheta_{\text {Geometrie }}, \mathrm{g}_{10}-\mathrm{g}_{12}\right.$ in Abb. 5) und Stoffeigenschaften ( $\delta \vartheta_{\text {Stoff }}, \mathrm{s}_{1}-\mathrm{s}_{9}$ in Abb. 5) sowie Randbedingungen $\left(\delta \vartheta_{\mathrm{RB}}, \mathrm{rb}_{19}-\mathrm{rb}_{23}\right.$ in Abb. 5 und Abb. 10 rechts), thermischen Kontakten bzw. Widerständen $\left(\delta \vartheta_{\text {therm. Kontakte, }} \mathrm{tk}_{1} 3-\mathrm{tk}_{18}\right.$ in Abb. 5) und numeri-

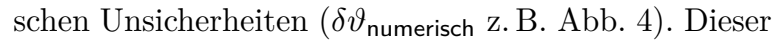
letzte Beitrag kommt zustande durch die automatische Zeitschrittsteuerung, die während der transienten Simulation in ANSYS zugelassen wird. Es wurde getrennt untersucht, um ihren möglichen systematischen Einfluss auf das Ergebnis besser ermitteln zu können. Die Auswahl von geringen festen Zeitschritten ist wegen der Wiederholung der Simulation für die Stichprobenberechnung nicht geeignet.

Der gesamte Term $\left(\delta \vartheta_{\text {Geometrie }}+\delta \vartheta_{\text {therm.Kontakte }}+\right.$ $\left.\delta \vartheta_{\mathrm{RB}}+\delta \vartheta_{\text {Stoff }}+\delta \vartheta_{\text {numerisch }}\right)$ entspricht der Reproduzierbarkeit von $\vartheta_{\mathrm{WP}_{\mathrm{s}}}$. Die einzelne Beiträge werden nicht getrennt ermittelt, sondern man erhält den gesamten Term aus der Stichprobenberechnung.

$\vartheta_{\text {Geometrie }}$ : Es wurden die Länge der einzelnen Fixpunktmaterialien in Abhängigkeit der Dichteänderung mit der Temperatur variiert ( $g_{10}$ für $\mathrm{In}, \mathrm{g}_{11}$ für $\mathrm{Sn}$ und $\mathrm{g}_{12}$ für $\mathrm{Zn}$ in Abb. 5 links).

$\delta \vartheta_{\text {therm. Kontakte: }}$ Die thermische Widerstände wurden einzeln untersucht, indem der Einfluss bei einem Temperatursprung auf die vom Temperatursensor gemessenen Temperatur mit einer maximalen definierten Luftspaltdicke von 0,25 $\mathrm{mm}$ und ohne thermischen Widerstand verglichen wurde. Sie wurden auf insgesamt 6 reduziert ( $\mathrm{tk}_{13}-\mathrm{tk}_{18}$ in Abb. 5 rechts).

$\delta \vartheta_{\mathrm{RB}}$ : Die Anfangstemperatur $\mathrm{rb}_{19}$, der Wärmeübergangskoeffizient $\mathrm{rb}_{20}$, die Temperatur bei $p_{1} \mathrm{rb}_{21}$ bzw. bei $p_{3} \mathrm{rb}_{22}$ und den maximalen Zeitschritt der Simulation $\mathrm{rb}_{23}$ wurden als Eingangsparameter angenommen.

$\delta \vartheta_{\text {Stoff }}:$ Die thermische Stoffeigenschaften $(\lambda, c, \rho$ oder $h)$ von acht im Systemaufbau präsenten Materialien wurde für $\vartheta_{90}$ jedes Fixpunktmaterial aus Literaturdaten interpoliert $[2-8,11-15,18,21-24,27-$ 30, 33, 34, 36, 37, 39-43, 45, 46]. Allen gefundenen Eigenschaften jeder Literaturquelle wurde die gleiche Wahrscheinlichkeit zugewiesen. Diese wurden der Einfachheit halber zu einer Temperaturleitfähigkeit ( $a$ [17]) pro Material kombiniert ( $\mathrm{s}_{1}$ bis $\mathrm{s}_{8}$ in Abb. 5 links). Luft wurde nicht berücksichtigt, weil im Temperaturbereich keine Unterschiede ihrer thermischen Eigenschaften gefunden wurden. Bei der Phasenumwandlung findet eine sprunghafte Änderung der Enthalpie $(H)$ statt. Diese Nichtlinearität der spezifischen Enthalpie $(h)$ wird in ANSYS für den Phasenübergang verwendet [38]. Die Fixpunkttemperatur weist eine Unsicherheit bezüglich der Konzentration von Verunreinigungen in den Fixpunktmaterialien auf [20], [32], dies wird zusätzlich als Eingangsparameter behandelt (s9 für In, Sn und Zn in Abb. 5 links). 

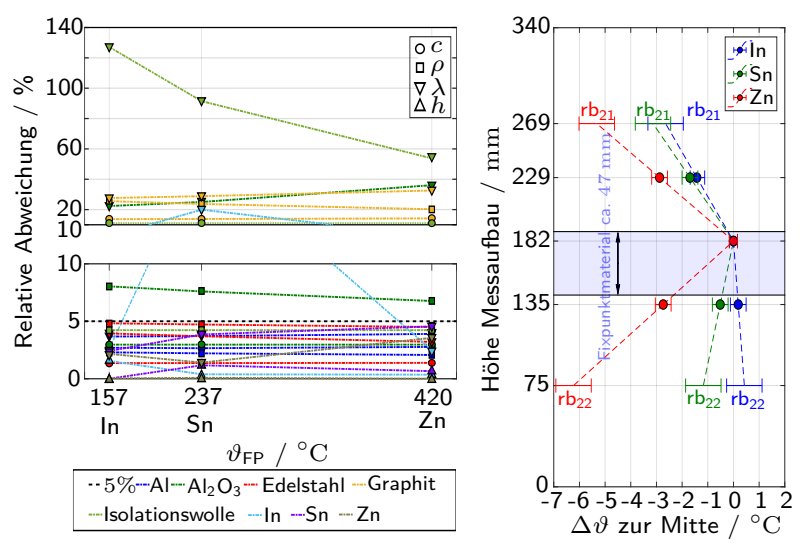

Abb. 10: Links: Relative Abweichung der Stoffeigenschaften. Rechts: Temperaturprofil bezüglich der Mitte der k-MFFPZ.

Abbildung 10 links zeigt die relative Abweichung der Stoffeigenschaften des Systemaufbaus. Für das Isolationsmaterial ist die relative Abweichung von $\lambda$ auffällig groß, vor allem bei niedrigen Temperaturen, da Literaturwerte in der Regel bei höheren Temperaturen $\left(600{ }^{\circ} \mathrm{C}\right.$ bis $1000^{\circ} \mathrm{C}$ ) angegeben werden. Für In ist die relative Abweichung von $\lambda$ nach seiner Phasenumwandlung deutlich größer als bei den anderen zwei Temperaturen, weil die gefundenen Daten entweder vor der Fixpunkttemperatur aufhören oder nach ihr anfangen. Die relative Abweichung aller thermischen Eigenschaften von Graphit und Aluminiumoxid ist relativ groß wegen der nicht genau bekannten Porosität der Materialien. Die Abweichung der spezifischen Enthalpie $h$ für die Fixpunktmaterialien wurde mit der Dichte $\rho$ berücksichtigt, weil nur eine Literaturquelle für diese Daten gefunden wurde [4]. Anhand der Graphik 10 links konnte festgestellt werden, dass gut bekannte Materialeigenschaften eine relative Abweichung von maximal $5 \%$ zeigen.

\section{Ergebnisse}

Fünf einzelne Simulationen mit unterschiedlich festgelegten Ofentemperaturen wurden für jedes Fixpunktmaterial durchgeführt (Manuelle Simulationen in Abb. 6). Daraus wurden mittels Ausgleichsrechnungen die Fixpunkttemperaturen $\left(\vartheta_{\text {fit }}\right.$ in Abb. 11) unter adiabatischen Bedingungen $\left(\Delta \vartheta_{\text {Ofen }}=0\right.$ in Abb. 11) ermittelt. Das Vertrauensintervall dieser Ausgleichsrechnung wurde durch das Vertrauensintervall oder die Prognosefähigkeit des Modells (Tab. 2) beschränkt bzw. verkleinert.

Zuletzt wurde das Unsicherheitsmodell eingesetzt und Mess- und Simulationsergebnisse wurden wieder verglichen

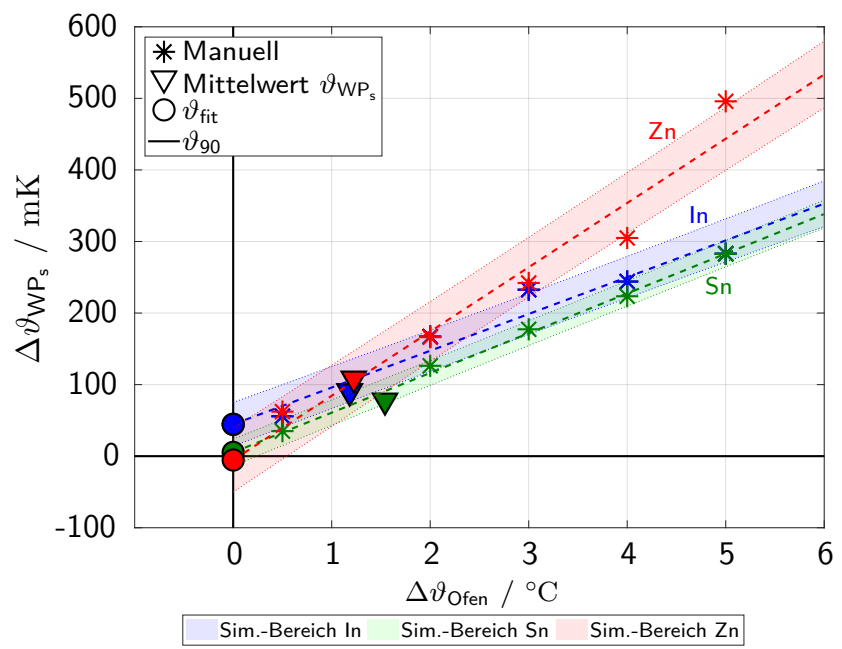

Abb. 11: Manuell durchgeführte Simulationen für die Ermittlung von $\vartheta_{\text {fit }}$ der drei Fixpunktmaterialien. Sim. Bereich zeigt das Vertrauensintervall jeder Ausgleichsrechnung.

(Abb. 1 und Gleichung 1). Da das berechnete Vertrauensintervall selber eine Unsicherheit hat $(67 \% \pm 12 \%, k=2$, Tab. 2) wurde der neue Vergleich durch Stichprobenberechnung mehrfach wiederholt. Daraus resultierte, dass die Wahrscheinlichkeit, mit der das Modell die Fixpunkttemperaturen aller 3 Fixpunktmaterialien unter adiabatischen Bedingungen vorhersagen kann, bei $64 \% \pm 16 \%, k=2$, liegt (Abb. 12).

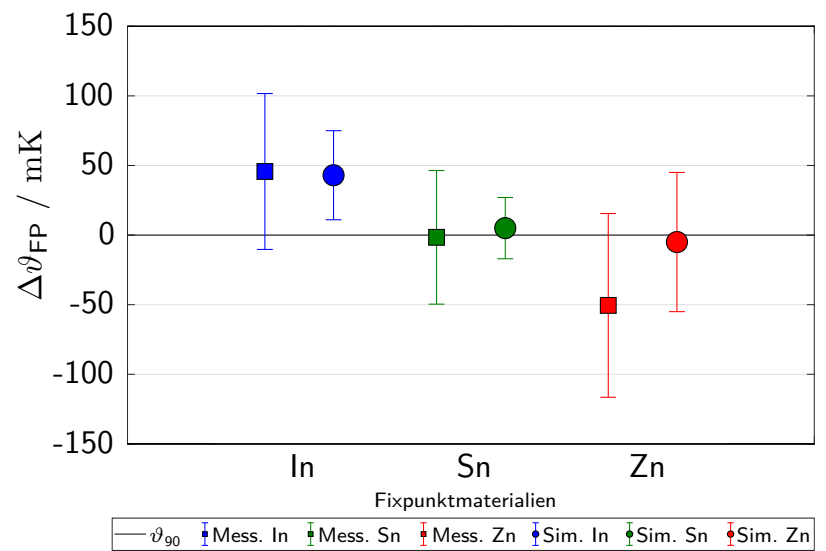

Abb. 12: $\vartheta_{\text {FP }}$ der Mess- und Simulationsergebnissen unter adiabatischen Bedingungen. Erweiterte Unsicherheiten, $k=2$. 


\section{Diskussion}

Eine Charakterisierung des Modells durch die Berechnung seiner Prognosefähigkeit erlaubt, genaueren Aussagen im Fall einer späteren Optimierung zu machen, weil die Grenzen des Modells besser bekannt sind. Man muss sich aber immer im Klaren sein, dass ein Modell nicht alle physikalischen Effekte abbilden kann, z.B der Effekt der im Fixpunktmaterial gelösten Verunreinigungen, die einen undeutlichen Anfang des Phasenüberganges verursachen oder die Bewegungen von noch festen Material während der Phasenumwandlung, was sich im Form von einem leichten Temperaturabfall beobachten lässt (Messung in Abb. 3). Eine Prognosefähigkeit von $100 \%$ wurde deswegen im hier berechneten Fall eine Übereinstimmung der Ergebnisse unter adiabatischen Bedingungen zwischen $70 \%$ und $95 \%$ bringen. Es muss hier zusätzlich beachtet werden, dass, wenn die Unsicherheit der Simulationsergebnisse größer als die der Messergebnisse wird, die Übereinstimmung von beiden wieder kleiner wird.

\section{Literatur}

[1] ANSYS Inc. Theory Reference for the Mechanical APDL and Mechanical Applications V12, 2009.

[2] M. J. Assael, A. E. Kalyva, K. D. Antoniadis, R. Michael Banish, I. Egry, J. Wu, E. Kaschnitz und W. A. Wakeham. Reference Data for the Density and Viscosity of Liquid Copper and Liquid Tin. Journal of Physical and Chemical Reference Data, 39(3):033105, 2010.

[3] M. J. Assael, I. J. Armyra, J. Brillo, S. V. Stankus, J. Wu und W. A. Wakeham. Reference Data for the Density and Viscosity of Liquid Cadmium, Cobalt, Gallium, Indium, Mercury, Silicon, Thallium, and Zinc. Journal of Physical and Chemical Reference Data, 41(3):033101, 2012.

[4] I. Barin. Thermochemical data of pure substances. VCH, New York, 1989.

[5] M. Binnewies und E. Milke. Thermochemical data of elements and compounds. Wiley-VCH, Weinheim [etc.], 1999.

[6] Bundesanstalt für Arbeitsschutz und Arbeitsmedizin. Technical Rules for Hazardous Substances TRGS 619: Substitute materials for aluminium silicate wool products, 2013.

[7] F. Cardarelli. Materials Handbook: A Concise Desktop Reference. Springer-Verlag, London, 2 Auflage, 2008.

[8] Deutsche Edelstahlwerke GmbH. Acidur 4541: Werkstoffdatenblatt X6CRNiTi18-10 1.4541, 2015.

[9] Dynardo GmbH. Methods for multidisciplinary optimization and robustness analysis, 2012.

[10] S. Ferson, W. L. Oberkampf und L. Ginzburg. Model validation and predictive capability for the thermal challenge problem. Computer Methods in Applied Mechanics and Engineering, 197(29 - 32):2408 - 2430, 2008.
[11] H. E. Friedrich und B. L. Mordike. Magnesium Technology. Springer-Verlag Berlin Heidelberg, [New York], 2006.

[12] T. Gancarz, W. Gąsior und H. Henein. Physicochemical Properties of Sb, Sn, Zn, and Sb-Sn System. International Journal of Thermophysics, 34(2):250-266, 2013.

[13] I. S. Grigoriev und E. Z. Meilikhov. Handbook of physical quantities. CRC Press, Boca Raton, Florida, 1997.

[14] Gruppo Lucefin S.P.A. Austenitic Stainless Steel 1.4541: Technical card 2018, 2018.

[15] C. Y. Ho, R. W. Powell und P. E. Liley. Thermal conductivity of the elements: A comprehensive review. J. Phys. Chem. Ref. Data, Suppl. 1, (3):1-796, 1974.

[16] M. Hohmann, S. Marin, M. Schalles und T. Fröhlich. Dry Block Calibrator with Improved Temperature Field and Integrated Fixed-Point Cells. International Journal of Thermophysics, 38(2):2360, 2017.

[17] F. P. Incropera und D. P. DeWitt. Fundamentals of heat and mass transfer. Wiley, New York and Chichester, 5th ed. Auflage, 2002.

[18] International Atomic Energy Agency. Thermophysical properties of materials for nuclear engineering: A tutorial and collection of data. International Atomic Energy Agency, Vienna, 2008.

[19] G. Krapf, M. Schalles und T. Fröhlich. Estimation of fixedpoint temperatures - A practical approach. Measurement, 44 (2):385-390, 2011.

[20] G. Krapf, H. Mammen, G. Blumröder und T. Fröhlich. Influence of impurities on the fixed-point temperature of zinc: Estimations by the SIE method and practical limitations. Measurement Science and Technology, 23(7):074022, 2012.

[21] H. Landolt, R. Börnstein, W. Martienssen und O. Madelung. Numerical data and functional relationships in science and technology: New series. Group 4, Physical chemistry. Vol. 19: Thermodynamic properties of inorganic materials, subvol. A: Pure substances. Springer, Berlin, 2000.

[22] D. R. Lide. CRC handbook of chemistry and physics: $A$ ready-reference book of chemical and physical data. CRC, Boca Raton, 83. ed. Auflage, 2002.

[23] M. Woite Sonderwerkstoffe Edelstähle. Werkstoff Nr.: 1.4541, 2012.

[24] O. Madelung und G. K. White, editors. Thermal Conductivity of Pure Metals and Alloys. Landolt-Börnstein - Group III Condensed Matter. Springer, Berlin, 1991.

[25] S. Marin, M. Hohmann, M. Schalles, G. Krapf und T. Fröhlich. Einsatzblock mit einer Mehrfachfixpunktzelle für einen Temperatur-Blockkalibrator. tm - Technisches Messen, 82 (7-8), 2015.

[26] S. Marin, M. Hohmann und T. Fröhlich. Small Multiple Fixed-Point Cell as Calibration Reference for a Dry Block Calibrator. International Journal of Thermophysics, 38(2): 2085, 2017.

[27] W. Martienssen und H. Warlimont. Springer Handbook of Condensed Matter and Materials Data. Springer, New York, 2005.

[28] W. Martienssen und H. Warlimont, editors. Metal Forming Data of Ferrous Alloys - deformation behaviour. LandoltBörnstein - Group VIII Advanced Materials and Technologies. Springer, Berlin, Heidelberg, 2009.

[29] Morgan Advanced Materials. Thermal Ceramic Products: Product data book: Kaowool 500 Grade, 2016. 
[30] Morgan Advanced Materials. Thermal Ceramic: Data sheet: Superwool Paper, 2016.

[31] W. L. Oberkampf und C. J. Roy. Verification and Validation in Scientific Computing. Cambridge University Press, 1 Auflage, 2010.

[32] J. V. Pearce, J. A. Gisby und P. P. M. Steur. Liquidus slopes of impurities in ITS-90 fixed points from the mercury point to the copper point in the low concentration limit. Metrologia, 53(4):1101-1114, 2016.

[33] M. V. Peralta-Martinez und W. A. Wakeham. Thermal Conductivity of Liquid Tin and Indium. International Journal of Thermophysics, 22(2):395-403, 2001.

[34] R. W. Powell, C. Y. Ho und P. E. Liley. Thermal Conductivity of Selected Materials. NSRDS-NBS. U.S. Gov. Print. Off, Washington, 1966.

[35] H. Preston-Thomas. The International Temperature Scale of 1990 (ITS-90). Metrologia, 27(1):3-10, 1990.

[36] Promat GmbH. Technisches Datenblatt ALSIFLEX: Produkte: 1230, 1260, 1400, 1430, 1600, 2017.

[37] Refrastraße S.A, subsidiaria de Keramische Technologie LLC. Catálogo de Productos: TEK Papier 1260, 2016.

[38] W. Schätzing, G. Müller, C. Groth und U. Stelzmann. FEM für Praktiker: Basiswissen und Arbeitsbeispiele zur FiniteElement-Methode mit dem FE-Programm ANSYS Rev. 5.5, Band 23 ; $44 ; 45$; 60 of Edition Expertsoft. Expert-verlag, Renningen-Malmsheim, verschiedene aufl. Auflage, 2000.

[39] SGL CARBON GmbH. Materialdaten SIGRAFINE R6650, 2018.

[40] J. F. Shackelford und R. H. Doremus. Ceramic and glass materials: Structure, properties and processing. Springer, New York, 2008.

[41] M. Spittel und T. Spittel. Steel symbol/number: X5crni1810/1.4301. In H. Warlimont, editor, Materials - Metal Forming Data of Ferrous Alloys - deformation behaviour. Landolt-Börnstein - Group VIII Advanced Materials and Technologies. Springer Berlin Heidelberg, Berlin, Heidelberg, 2009.

[42] D. J. Steinberg. A simple relationship between the temperature dependence of the density of liquid metals and their boiling temperatures. Metallurgical Transactions, 5(6): 1341-1343, 1974.

[43] VDI e.V. VDI-Wärmeatlas: 11., bearbeitete und erweiterte Auflage. Springer, Berlin, Heidelberg, Dordrecht, London, New York, 2013.

[44] M. Voigt. Probabilistische Simulation des strukturmechanischen Verhaltens von Turbinenschaufeln. PhD thesis, Universität Dresden, 2009.

[45] W. Weißbach, M. Dahms und C. Jaroschek. Metallische Werkstoffe. In W. Weißbach, M. Dahms und C. Jaroschek, editors, Werkstoffkunde, S. 25-109. Springer Fachmedien Wiesbaden, Wiesbaden, 2015.

[46] E. Yamasue, M. Susa, H. Fukuyama und K. Nagata. Deviation from Wiedemann-Franz Law for the Thermal Conductivity of Liquid Tin and Lead at Elevated Temperature. International Journal of Thermophysics, 24(3):713-730, 2003. 